\title{
Anesthetic Goncerns in Patients with Retinopathy of Prematurity
}

\section{Charu Neema}

From the Department of Anaesthesia and Critical Care, Sri Aurobindo Institute of Medical Sciences, Indore, India.

\section{Abstract:}

Retinopathy of prematurity (ROP) is a disorder of the developing retina of low birth weight preterm infants, that may be the potential cause of blindness in such infants. Peripheral retinal ablation with diode laser is the preferred method for treatment of severe ROP. Examination and surgery (laser ablation and cryotherapy) for ROP are known to be extremely stressful and probably painful to the neonate. Topical anesthetic pretreatment can reduce the pain response but is not effective in all neonates. Thus, even for simple examination of the eye, topical anesthetic alone may be insufficient. If we accept that laser therapy is at least stressful, if not significantly painful, it can be seen that these babies require more than minimal sedation. The case reports mentioned below describe two different ways of the anesthetic management of infants with ROP. The article focuses on the various anesthetic methods available, and anesthetic concerns in such infants.

Key words: Retinopathy of Prematurity, Retina, Blindness, Cryotherapy, Cryosurgery, Infant.

\section{Introduction}

Retinopathy of prematurity (ROP) is a vasoproliferative disorder of the retina among premature babies. ROP begins to develop between 32 and 34 weeks after conception, regardless of gestational age at delivery [1]. Peripheral retinal ablation of avascular retina can be done by either cryotherapy or diode laser. Diode laser ablation has replaced cryotherapy due to lower rate of postoperative ocular and systemic complications. Laser therapy for ROP is known to be stressful and probably painful to the neonate. It is accepted that the premature baby is capable of feeling pain and repeated and significant painful stimuli can result in significant morbidity as a result of prolonged bradycardia and apneic episodes [2-4]. No consensus on the optimum method of anesthesia for the treatment of ROP currently exists. The purpose of this article is to consider various aspects in the perioperative care of the patients with ROP, discuss the different anesthetic methods and highlight the problems associated with providing safe and optimal care for premature babies requiring anesthesia and surgery.

Corresponding Author: Dr.Charu Neema

Email: charu.neema@gmail.com

Received: January 10, 2013 | Accepted: January 17, 2013 | Published Online: February 5, 2013

This is an Open Access article distributed under the terms of the Creative Commons Attribution License (creativecommons.org/licenses/by/3.0)

Conflict of interest: None declared | Source of funding: Nil | DOl: http://dx.doi.org/10.17659/01.2013.0015 


\section{Case Report}

Case 1: A 2 month old premature male born at 30 weeks post conception, weighing $2.5 \mathrm{~kg}$ and diagnosed with ROP was posted for laser ablation. Patient had history of respiratory distress at birth for which ventilatory support was given to the baby for 7 days. All routine investigations were within normal limits. After maintaining standard preoperative guidelines, infant was premedicated with $0.1 \mathrm{mg}$ of intravenous atropine. Anesthesia was induced with ketamine $5 \mathrm{mg}$ and atracurium $1.25 \mathrm{mg}$ by intravenous route and baby was intubated. Anesthesia was maintained with oxygen and air mixture and sevoflurane. The procedure lasted for 30 minutes after which neuromuscular blockade was reversed and patient was extubated. Baby stood the procedure well and was shifted to post anesthesia care unit.

Case 2: A one and a half month old male child born at 32 weeks gestational age weighing $2 \mathrm{~kg}$ diagnosed with ROP was posted for laser retinal ablation. Patient had history of jaundice and apneic spells at birth. All routine investigations were within normal limits. After keeping the infant nil per orally for 4 hours and securing intravenous access, child was premedicated with $0.1 \mathrm{mg}$ atropine by intravenous route. Anesthesia was induced with intravenous ketamine $4 \mathrm{mg}$ and maintained with sevoflurane and oxygen air mixture. Monitoring of vitals was done throughout the procedure and all measures to avoid hypothermia were taken. The child was kept on spontaneous respiration on Jackson Rees circuit. The whole course of anesthesia was uneventful for the procedure which lasted for 20 minutes. At the end of procedure the child was shifted to post anesthesia care unit.

\section{Discussion}

During the acute first phase of ROP, the normal vasculogenesis of the retina is disturbed by the relative hyperoxia of the extrauterine environment. This causes vaso-obliteration and non-vascularization of some areas of the anterior retina [5]. The subsequent hypoxia causes a second chronic phase, characterized by the proliferation of vascular and glial cells arteriovenous shunt formation, occasionally leading to involution or permanent cicatricial changes and visual impairment [6,7]. Studies from India reporting incidence of ROP provide interesting insights. Although screening criteria differ across different units and time-periods, overall incidence of ROP varies from $20 \%$ to $52 \%$, with more recent studies reporting lower rates of ROP ranging from $20 \%$ to $30 \%$ [8-16]. Prematurity is the single most important risk factor. Exposure to a $\mathrm{PaO}_{2}$ higher than $80 \mathrm{mmHg}$ for prolonged periods (premature infants 500 to 1300 grams) may be associated with an increased incidence and severity of ROP. The risk of retinopathy is inversely related to birth weight, with significant risk in infants weighing less than 1500 grams [17]. Other risk factors which increase the probability of developing ROP are anaemia needing blood transfusion, sepsis and apnea. Neonates and infants with ROP are prone to prematurity-related morbidities including respiratory distress syndrome, intraventricular haemorrhage, periventricular leukomalacia, patent ductus arteriosus and necrotising enterocolitis.

Preoperative evaluation: A thorough preoperative assessment should be carried out and any potential risk factors associated with anesthesia are discussed with the parents. Fasting guidelines in neonates and infants should be followed.

Pre-anesthetic medication: Baby should be started on intravenous fluids, and put on cardio-respiratory monitor. 
Neonates do not usually require sedation. Atropine, can be given when required, either orally or, less frequently, intramuscular. It is used for vagolytic activity and to decrease airway secretions. Atropine may also be given intravenously on induction, either routinely, or if bradycardia occurs. Ametop can be used as a local anesthetic cream to assist intravenou scannulation. EMLA is not recommended for babies $<12$ months old as metabolism of prilocaine results in methaemoglobinaemia. Dilatation of pupil is done by using $0.5 \%$ tropicamide and $2.5 \%$ phenylephrine.

Intraoperative considerations: Topical anesthesia alone provides insufficient analgesia for ROP treatment and should not be used. The procedure can be carried out under general anesthesia or under sedation. Routine monitoring should be used and is specifically designed for babies. This includes electrocardiogram, non-invasive blood pressure, capnography, pulse oximetry, temperature, and neuromuscular monitoring. The pharmacokinetics of drugs in preterm infants is not fully understood but smaller doses of anesthetic drugs are usually required in preterm infants compared to term infants and older children and their effects last longer due to low clearance rates and longer elimination half-lives [18]. Sevoflurane is the inhalational induction agent of choice in most neonates. It has a rapid onset with cardiovascular stability. Thiopentone remains a useful agent, but propofol is unlicensed in this age group and there is little information about this agent in neonates. Ketamine is used occasionally. The functional residual capacity in neonates and infants is reduced and there is risk of ventilation/perfusion mismatch. Airway can be secured with an endotracheal tube, whilst the size one laryngeal mask airway provides a convenient airway it is liable to dislodgement and longer procedures are poorly tolerated. Atracurium is the most useful muscle relaxant as neonatal enzyme systems are immature with unpredictable metabolism of other relaxant drugs. Maintenance of anesthesia is with oxygen in either nitrous oxide or air. It is important to avoid administration of excessive concentrations of oxygen in premature babies. Key anesthetic considerations are (i) inspired oxygen concentration that should be adjusted to avoid hyperoxia, (ii) hemodynamic parameters that should be kept stable and (iii) prevention of hypothermia by using adequate measures to keep the infants warm [18]. Both the eyes can be treated at the same sitting time unless contraindicated by instability of the baby.

Anesthetic methods: In a study by Haigh et al, premature babies who received topical local anesthetic eye drops alone before cryotherapy suffered severe and recurrent cardiorespiratory complications. However, the babies who were managed with controlled ventilation and either sedative or anesthetic drugs had significantly fewer complications [19]. Although the application of diode laser is less painful, there has to be adequate immobilization of the patient to provide a correct focus of the spot. In the first case, the baby was given general anesthesia with muscle relaxant, while in the second case local anesthesia was supplemented with intravenous ketamine and oxygen-sevoflurane mixture with baby breathing spontaneously. Ferrer et al obtained sedation with inhaled anesthetic agents combined with topical anesthesia for laser ablation [20]. A survey conducted by Chen and his colleagues reveals considerable variation in practice among UK ophthalmologists regarding the anesthetic methods employed in the laser treatment of ROP, and their beliefs regarding the systemic stress associated with treatment. In Chen's article $50 \%$ of respondents reported the use of intubation and ventilation during laser treatment. The remainder received various types of sedation without airway protection or ventilator support. Oral sedation combined with topical anesthesia, rectal chloral hydrate and paracetamol combined with topical anesthesia, intravenous ketamine combined with topical anesthesia, and subtenon's local anesthesia were used by one $(3 \%)$ respondent each. There were no ophthalmologists using subconjunctival or topical anesthetic or treating without anesthesia [21]. Lyon et al used ketamine sedation and found that it 
produces few intra- or postoperative complications for the infant, while providing satisfactory conditions for the treatment of ROP [22]. A study conducted by Kirwan et al demonstrates that morphine analgesia can be used as an alternative to general anesthesia [23]. It can be seen that these babies require more than minimal sedation. They actually require deep sedation and analgesia. Regardless of the intended level of sedation or route of administration of sedative, a pediatric patient and particularly a neonate may move easily from a level of sedation to obtundation with the loss of protective reflexes [24]. So airway protection ensures better safety and avoids complications like hypoxia and bradycardia during the procedure. Laryngeal mask airway is a safe and easy to use alternative for airway management during laser photocoagulation procedure in infants with ROP [25]. According to Takauchi et al general anesthesia with volatile anesthetics appeared to be superior in actual outcome to ketamine anesthesia among the patients with high risk scores [26].

Post-operative care: If condition permits, oral feeds can be started shortly after the procedure. Premature babies, especially those with chronic lung diseasemay have apneic episodes and, should be carefully monitored for 48-72 hours after the procedure. Antibiotic drops (such as chloramphenicol) should be instilled 6-8 hourly for 2-3 days.

\section{Conclusion}

If the peripheral retinal ablation is carried out under local anesthesia only erratic swings in heart rate and blood pressure and hypoxia can result in significant morbidity. The most important issue is to ensure the babies safety as these babies are premature and due to the various associated comorbidities, controlled intubation and ventilation or sedation combined with local anesthesia can be the main stay in the anesthetic management.

\section{References}

1. Flynn JT. The premature retina: a model for the in vivo study of molecular genetics? Eye.1992;6:161165.

2. Anand KJ, Carr DB. The neuroanatomy, neurophysiology and neurochemistry of pain, stress and analgesia in newborns and children. Pediatr Clin N Am. 1989;36:795-822.

3. Perreault T, Fraser-Askin D, Listan R. Pain in the neonate. Paediatr Child Health. 1997;2:201-209.

4. Anand KJ. Clinical importance of pain and stress in preterm neonates. Biol Neonate. 1998;73:1-9.

5. Kushner BJ, Essner D, Cohen IJ, Flynn JT. Retrolental Fibroplasia. II. Pathologic correlation. Arch Ophthalmol. 1977;95:29-38.

6. Chan-Ling T, Tout S, Hollander H, Stone J. Vascular changes and their mechanisms in the feline model of retinopathy of prematurity. Invest Ophthalmol Vis Sci. 1992;33:21 28-2147.

7. Chan-Ling T, Gock B, Stone J. The effect of oxygen on vasoformative cell division. Evidence that 'physiological hypoxia' is the stimulus for normal retinal vasculogenesis. Invest Ophthalmol Vis Sci. 1995;36:1201-1214.

8. Chaudhari S, Patwardhan V, Vaidya U, Kadam S, Kamat A. Retinopathy of prematurity in a tertiary care center--incidence, risk factors and outcome. Indian Pediatr. 2009;46:219-224.

9. Charan R, Dogra MR, Gupta A, Narang A. The incidence of retinopathy of prematurity in a neonatal care unit. Indian J Ophthalmol. 1995;43:1 23-126. 
10. Gupta VP, Dhaliwal U, Sharma R, Gupta P, Rohatgi J. Retinopathy of prematurity-risk factors. Indian J Pediatr. 2004;71:887-892.

11. Maheshwari R, Kumar H, Paul VK, Singh M, Deorari AK, Tiwari HK. Incidence and risk factors of retinopathy of prematurity in a tertiary care newborn unit in New Delhi. Natl Med J India. 1996;9:211-214.

12. Aggarwal R, Deorari AK, Azad RV, Kumar H, Talwar D, Sethi Al. Changing profile of retinopathy of prematurity. J Trop Pediatr. 2002;48:239-242.

13. Dutta S, Narang S, Narang A, Dogra M, Gupta A. Risk factors of threshold retinopathy of prematurity. Indian Pediatr. 2004;41:665-671.

14. RekhaS, BattuRR. Retinopathy of prematurity: incidence and risk factors. Indian Pediatr. 1996;33:999-1003.

15. Gopal L, Sharma T, Ramachandran S, Shanmugasundaram R, Asha V. Retinopathy ofprematurity: a study. Indian J Ophthalmol. 1995;43:59-61.

16. Varughese S, Jain S, Gupta N, Singh S, Tyagi V, Puliyel JM. Magnitude of the problem of retinopathy of prematurity, experience in a large maternity unit with a medium size level-3 nursery. Indian J Ophthalmol. $2001 ; 49: 187-188$.

17. Phelps DL. Retinopathy of prematurity.NEngl J Med. 1992;326:1078-1080.

18. Kinouchi K. Anaesthetic considerations for the management of very low and extremely low birth weight infants. Best Pract Res Clin Anaesthesiol. 2004; 18:273-290.

19. Haigh PM, Chiswick ML, O'Donoghue EP. Retinopathy of prematurity: systemic complications associated with different anaesthetic techniques at treatment. Br J Ophthalmol. 1997;81:283-287.

20. Ferrer Novella C, González Viejo I, Oro Fraile J, Mayoral López F, DiesteMarcial M. New anaesthetic technique in diode laser treatment of retinopathy of prematurity. An Pediatr (Barc). 2008;68:576-580.

21. Chen SD, Sundaram V, Wilkinson A, Patel CK. Variation in anaesthesia for the laser treatment of retinopathy of prematurity--a survey of ophthalmologists in the UK. Eye (Lond). 2007;21:1033-1036.

22. Lyon F, Dabbs T, O'Meara M. Ketamine sedation during the treatment of retinopathy of prematurity. Eye (Lond). 2008;22:684-686.

23. Kirwan C, O'Keefe M, Prendergast M, Twomey A, Murphy J. Morphine analgesia as an alternative to general anaesthesia during laser treatment of retinopathy of prematurity. Acta Ophthalmol Scand. 2007;85:644-647.

24. Hartrey R. Anaesthesia for the laser treatment of neonates with retinopathy of prematurity. Eye. 2007;21:1025-1027; doi:10.1038/sj.eye.6702502

25. Gunenc F, Kuvaki B, lyilikci L, Gokmen N, Yaman A, Gokel E. Use of laryngeal mask airway in anesthesia for treatment of retinopathy of prematurity. Saudi Med J. 2011 ;32:1 127-1132.

26. Takauchi Y, Inamori N, Ohhashi Y, Tanigami H, Nishimura M, Fukumitsu K, Tashiro C. Respiratory outcome in extremely premature infants following general anesthesia. Masui. 1993;42:529-533. 